\title{
Realization of Curved Circular Nanotubes Using In Situ Monitored Self-Assembly
}

\author{
Zihao Lin, Chunhui Dai, and Jeong-Hyun Cho* \\ Department of Electrical and Computer Engineering, University of Minnesota, \\ Minneapolis, Minnesota 55455, United States \\ *Email:jcho@umn.edu
}

\section{List of Contents}

Fabrication of Sealed Curved Circular Nanofluidic Channel ----------------------------2

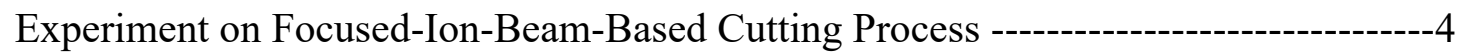

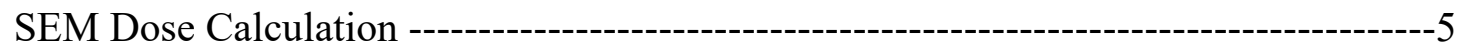

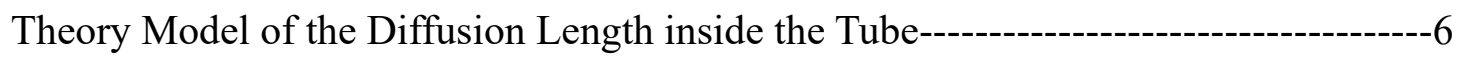

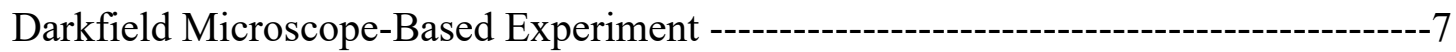

Simulation Model Design Description ----------------------------------------------------------8

Numerical Simulation of Light Scattering Effect Based on the Sample Under Darkfield

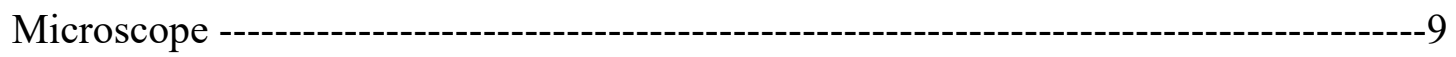

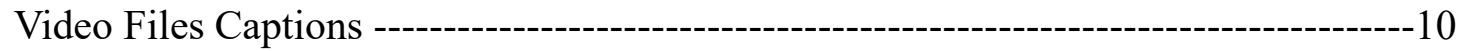

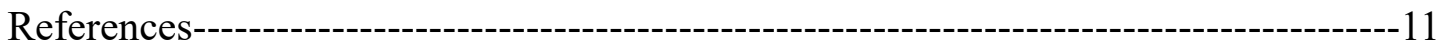




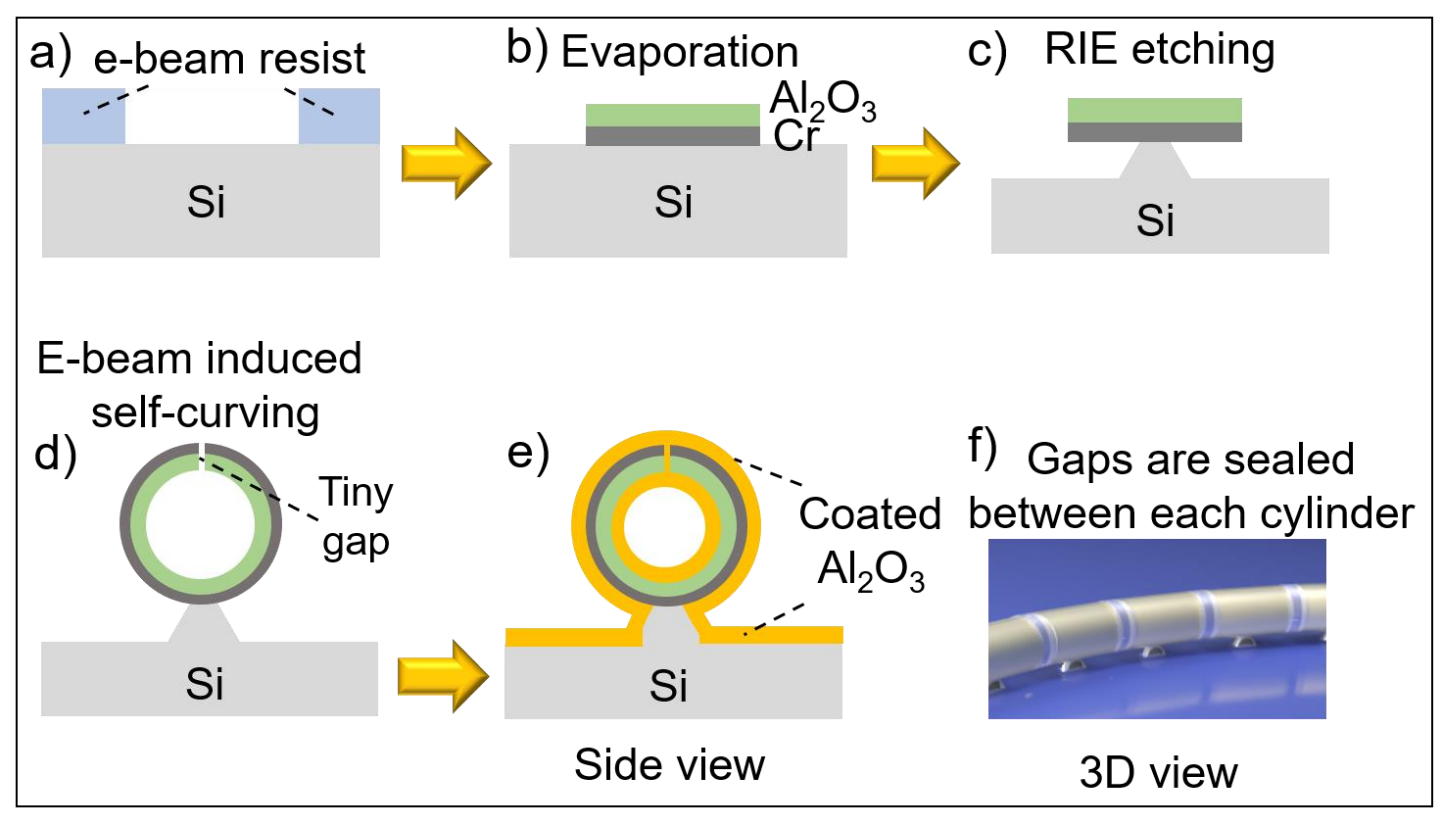

Figure S1. Fabrication procedures of realizing curved 3D circular nanotubes.

The fabrication procedures of realizing curved 3D circular nanotubes are shown in Figure S1. Basic cleaning process was used for Si chip in advance. PMMA A2 was spun on the chip at $3000 \mathrm{rpm}$, forming an about $150 \mathrm{~nm}$ PMMA layer followed by baking process at $180^{\circ} \mathrm{C}$ for $3 \mathrm{~min}$. Then, an electron beam lithography system (Vistec EBPG 5000+) was used to define 2D patterns in a width of $1.1 \mu \mathrm{m}$ ( $1 \mathrm{nA}$ diameter with a dose of $1300 \mu \mathrm{C} / \mathrm{cm}^{2}$, Figure S1a). The gaps between each pair of two patterns were $53 \mathrm{~nm}$ and $79 \mathrm{~nm}$ at the inner and outer sides based on our careful design to avoid collision during curving. The sample was then immersed under developer (MIBK: IPA=1:3) for 50 seconds. An e-beam evaporator (RME-E2000) was used to deposit 2 $\mathrm{nm} \mathrm{Cr}$ and $3 \mathrm{~nm} \mathrm{Al}_{2} \mathrm{O}_{3}$ to form a bilayer structure under the vacuum of $2.0 \times 10^{-7}$ Torr followed by liftoff process (Figure S1b). A reactive ion etching (RIE) system (STS 320) was used to remove the Si underneath $2 \mathrm{D}$ patterns until about $100 \mathrm{~nm}$ width remained 
(Figure $\mathrm{S} 1 \mathrm{c}$ ). During the etching process, the $\mathrm{CF}_{4}$ and $\mathrm{O}_{2}$ were flowed into the chamber at flow rates of $12 \mathrm{sccm}$ and $1 \mathrm{sccm}$, respectively. The etching process was conducted at a fixed pressure of $80 \mathrm{mTorr}$ and power of $20 \mathrm{~W}$ for $26 \mathrm{mins}$. To minimize the effect of ion bombardment during the RIE process, the sample was placed facing the bottom of the RIE chamber. The sample was exposed to electron beam irradiation with 12.6 $\mathrm{nA}$ beam current and $20 \mathrm{kV}$ bias voltage in a focus ion beam (FIB) system (dual-beam FIB/SEM, FEI Helios G4 UX, vacuum under $2 \times 10^{-4} \mathrm{~Pa}$ ). Electron beam in the system acted as a source for inducing the self-assembly process (Figure S1d). During the curving process, a problem usually occurred is half of the pattern was under irradiation while another half was not which brings a twisted 3D shape. To solve this problem, electron beam was quickly glanced from the first pattern to the end one and then back and forth to ensure that all the patterns were curved perfectly. Patterns stop curving when the boundary touches with each other. A thermal atomic layer deposition (ALD) system (Savannah, thermal ALD, $200^{\circ} \mathrm{C}, 600$ cycles) was used to completely encapsulate the gaps generated after self-assembly process by growing $\mathrm{Al}_{2} \mathrm{O}_{3}$ layer on tubes with Trimethylaluminum [TMA] and water vapor as precursors (Figure S1e,f). 


\section{Experiment on Focused-Ion-Beam-Based Cutting Process}

A tube with diameter of $\sim 50 \mathrm{~nm}$ and length of about $4 \mu \mathrm{m}$ was made in advance after sealing with ALD. After the vacuum was stable in FIB system, both electron beam and ion beam position were first calibrated and focused on the same position as the stage tilted to 52 degree. Pt needle was inserted at the center of the tube position and injected with $1 \mu \mathrm{m}$ thickness protective layer. Cutting area was drawn with a thickness of 1.5 $\mu \mathrm{m}$ and Regular Cross Section Pattern (RCSP) function was used to cut the area with $30.0 \mathrm{kV}$ voltage and $1.2 \mathrm{nA}$ current. In order to get a smooth surface of the cross section,

Cleaning Cross Section Pattern (CCSP) function was used to cut again with a decreased current at $90 \mathrm{pA}$. The SEM window was quickly activated and captured the photo of cross section area at the center of the tube. 
The dose calculation was based on a previous literature ${ }^{\mathrm{R} 1}$. The equation can be written as:

$$
\text { Dose }=\frac{\text { beam current } \cdot \text { irradiation time }}{\text { charge carried by the electron } \cdot \text { irradiated area }}
$$

Thus, the value of electron dose is determined by electron beam current, irradiation time and the irradiated area. The electron beam current used for curving was fixed at $3.2 \mathrm{nA}$, and the irradiated area was $22.5 \times 15.8 \mu \mathrm{m} \times \mu \mathrm{m}$. So, the dose is mainly governed by the irradiation time. As the time changes, the dose varies with the time. 


\section{Theory Model of the Diffusion Length inside the Tube}

According to a previous work ${ }^{\mathrm{R} 2}$, the mathematical equation based on Monte Carlo simulation to get the minimum exposure time to achieve the uniform deposition in the high aspect ratio nanotubes is given as:

$$
t=2.3 \times 10^{-7} p^{-1} m^{1 / 2} \Gamma(L / d)^{2}
$$

where $t$ is the exposure time in seconds, $P$ is the reactant pressure in Torr. $m$ is the mass of the reactant molecule (TMA), $\Gamma$ is the density of ALD reactive sites and $L / D$ is the aspect ratio of the tube. Based on the settings, $m$ is 72 in amu, $\Gamma$ is 0.2391 and $L / d$ for sealing curved circular tube with length of $90 \mu \mathrm{m}$ and diameter of $220 \mathrm{~nm}$ (for Figure 5a-d purpose) in final is about 225:1. Based on the equation, an exposure time $10 \mathrm{~s}$ is long enough to satisfy the equation. More scrupulously, this predicted exposure time is only valid in diffusion-limited region, where the reactive sticking coefficient should be much larger than the hopping coefficient ${ }^{\mathrm{R} 3}$. The sticking coefficient ${ }^{\mathrm{R} 4}$ for $\mathrm{Al}_{2} \mathrm{O}_{3}$ coating by ALD is $S=1 \times 10^{-3}$ while the hopping coefficient can be calculated as $H=16(d / L)^{2}$, the calculation result shows that $S$ is much larger than $H$, which is $6.4 \times 10^{-5}$. So, the predicted exposure time is reasonable. Similarly, for a $4 \mu \mathrm{m}$ tube with diameter about $10 \mathrm{~nm}$, the $H$ is $4 \times 10^{-4}$ which can also be considered in a safe range in diffusionlimited region. 


\section{Darkfield Microscope-Based Experiment}

After the sealing process, the sample was placed on the stage of an optical microscope (Nikon Eclipse LV150N, 20×objective, NA=0.45). A switch was adjusted from bright field to dark field mode. IPA was filled in syringe with a needle. Microscope camera is DS-Fi3 (Frame Rate: $16 \mathrm{fps}$ ). During the dropping process, since the width of the needle holder is larger than the distance between lens and sample, we switched lens to $10 \times 0.30$ NA for providing enough distance to drop IPA on the sample and then switched back. After dropping only one drop of IPA, the sample was immersed in liquid and then dried out, following by IPA in the tube dried out as well. The same experiment procedure was repeated for more than 10 times for the same sample, and it was confirmed that the sample was still robust and was not damaged since the same color changing behavior repeated for both straight and curved tube. The video provided in SI Video 1, 2 is by zooming in 16 times, 70 times of the original captured videos, respectively for a better visualization. 


\section{- Simulation Model Design Description}

A 2D refractive index monitor (x-height direction) was placed at the center of model $(\mathrm{z}=0)$, the result is shown in Figure $\mathrm{S} 2$, different colors show different refractive index of different materials. The red color represents the silicon, and the formation of arc structure is due to RIE etching process. Different mesh order and homemade script file was used to build this arc structure while only leaving about $100 \mathrm{~nm} \mathrm{Si}$ attached with the tube. All the silicon was covered by a $60 \mathrm{~nm} \mathrm{Al}_{2} \mathrm{O}_{3}$ due to ALD process. The color inside the tube represents the refractive index of IPA with a diameter of $220 \mathrm{~nm}$ after calculation carefully. The distance between tube and the substrate was set as about 900 nm after using Alpha stepper measurement. The length of tube which is not shown in Figure $\mathrm{S} 2$ is $1200 \mathrm{~nm}$ based on our 2D pattern design and the length of Si (z direction) attached under the tube is $400 \mathrm{~nm}$. The model is placed in atmosphere and illuminated by TFSF light source along y directions.

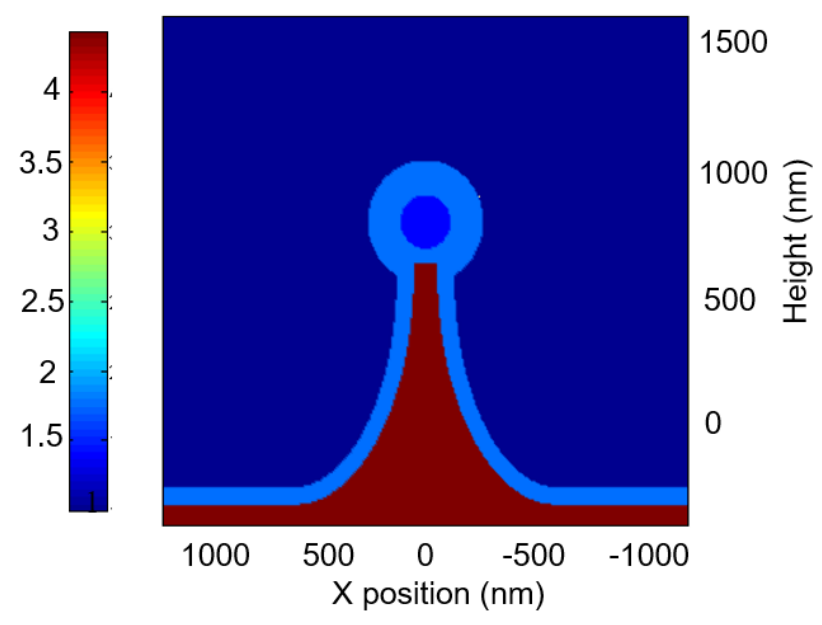

Figure S2. Refractive index monitor shows the simulation configuration (wavelength at $480 \mathrm{~nm}$ ). 
Numerical Simulation of Light Scattering Effect Based on the Sample Under Darkfield Microscope

A 3D simulation was performed in Ansys Lumerical 2020 R2.1 FDTD Solutions ${ }^{\mathrm{R} 5}$. All boundaries were set as perfect matching layer (PML) conditions with simulation time of $1000 \mathrm{fs}$ and auto shutoff at $1 \mathrm{e}^{-5}$. The incident light is injected from air side using a total-filed scattered-field (TFSF) light source with $27^{\circ}$ angle calculated by $N A$ of the objective. The light source is based on the real tungsten halogen lamp spectrum which was reported previously ${ }^{\mathrm{R} 6}$ and illumination spectrum in the visible light range was imported and controlled by home-made script file. A monitor was set at the back of the source to receive back scattered light. Darkfield experiment was performed under unpolarized light. Based on the setting suggestions from official ANSYS Lumerical website $^{\mathrm{R} 7}$, two light sources with TE and TM polarization direction illuminate the structure, respectively and the spectrum can be obtained by averaging the result from both polarizations. Considering the limitation of computational capabilities, the mesh size was set to be $4 \mathrm{~nm}$ along the light propagation direction (x- and $\mathrm{y}-$ axes) and $6 \mathrm{~nm}$ along another direction. The optical parameters of IPA were based on the previous literature $^{\mathrm{R} 8}$ while all other materials were picked from Palik in the material library of the software. Each wavelength of the light source was swept from the start to the end using Sweep function in the software. Data analysis was performed to normalize to the maximum intensity value of the spectra. 


\section{Video Files Captions}

\section{Video S1:}

The real-time video of an IPA evaporation process inside the curved tube under darkfield microscope. (MP4)

\section{Video S2:}

The real-time video of an IPA evaporation process inside the straight tube under darkfield microscope. (MP4) 


\section{$\underline{\text { Supplementary References }}$}

(R1) Mao, Y.; Zheng, Y.; Li, C.; Guo, L.; Pan, Y.; Zhu, R.; Xu, J.; Zhang, W.; Wu, W. Programmable Bidirectional Folding of Metallic Thin Films for 3D Chiral Optical Antennas. Adv. Mater. 2017, 29, 1606482.

(R2) Elam, J.W.; Routkevitch, D.; Mardilovich, P.P.; George, S.M. 2003.

Conformal Coating on Ultrahigh-Aspect-Ratio Nanopores of Anodic Alumina by Atomic Layer Deposition. Chem. Mater. 2003, 15, 3507-3517.

(R3) Zazpe, R.; Knaut, M.; Sopha, H.; Hromadko, L.; Albert, M.; Prikryl, J.;

Gartnerova, V.; Bartha, J.W.; Macak, J.M. Atomic Layer Deposition for Coating of High Aspect Ratio $\mathrm{TiO}_{2}$ Nanotube Layers. Langmuir 2016, 32, 10551-10558.

(R4) Elam, J.W.; Groner, M.D.; George, S.M. 2002. Viscous Flow Reactor with Quartz Crystal Microbalance for Thin Film Growth by Atomic Layer Deposition. Rev. Sci. Instrum. 2002, 73, 2981-2987.

(R5) ANSYS/LUMERICAL Home Page. https://www.lumerical.com/products/fdtd/ (accessed 2021-12-23).

(R6) Thoms, L.-J.; Girwidz, R. Experimenting from a Distance: Optical Spectrometry Via the Internet. MPTLAt: Madrid 2013, 18.

(R7) https://support.lumerical.com/hc/en-us/articles/360042703433-Mie-scatteringFDTD- (accessed 2021-12-23).

(R8) Sani, E.; Dell'Oro, A. Spectral Optical Constants of Ethanol and Isopropanol from Ultraviolet to Far Infrared. Opt. Mater. 2016, 60, 137-141. 